\title{
Una experiencia de formación: la Educación basada en evidencias
}

\author{
Magda Campillo Labrandero, Maura Pompa Mansilla, \\ Flora Beatriz Hernández Carrillo y Melchor Sánchez Mendiola
}

\begin{abstract}
Resumen
La Educación basada en evidencias (EBE) busca que los docentes y otros profesionales involucrados en la educación informen sus decisiones y acciones con los hallazgos encontrados en la literatura científica publicada en el campo educativo. En el presente artículo se comparte la experiencia de los cursos EBE, que desde 2016 ofrece la Coordinación de Desarrollo Educativo e Innovación Curricular (CODEIC) a los docentes de la Universidad Nacional Autónoma de México (UNAM). También se describe cómo se conformó la comunidad de práctica ceBe (Comunidad de Educación Basada en Evidencias) así como las actividades que realizan sus miembros. Tanto los cursos EBE Como la CEBE son experiencias de formación docente novedosas que han vinculado el arte de la enseñanza con los avances científicos en el campo de estudio de la educación.
\end{abstract}

Palabras clave: educación basada en evidencias, comunidades de práctica, formación docente.

\section{A tRAINING EXPERIENCE: EVIDENCE-BASED EDUCATION}

\begin{abstract}
Evidence-based education seeks to inform teachers and other educational agents' decisions and actions by the findings of scientific literature regarding education. The purpose of this article is to share our experience in developing and teaching evidence-based education courses to unAm faculty. These courses have been imparted since 2016 at the Coordinación de Desarrollo Educativo e Innovación Curricular (CODEIC). We also describe how a community of practice (CEBE) was created, as well as the activities held within such community. These courses and the community of practice are novel teaching training experiences that have linked the art of teaching with scientific discoveries in the field of education.
\end{abstract}

Keywords: evidence-based education, communities of practice, teacher training.

Dol: http://doi.org/10.22201/codeic.16076079e.2019.v20n6.a6 
"Una experiencia de formación: la Educación basada en evidencias"

Magda Campillo Labrandero, Maura Pompa Mansilla,

Flora Beatriz Hernández Carrillo y Melchor Sánchez Mendiola

Vol. 20, Núm. 6, noviembre-diciembre 2019

Revista Digital Universitaria

\section{Magda Campillo Labrandero}

mcl@unam.mx

Licenciada, maestra y doctora en Psicología educativa. Docente de educación superior y posgrado. Obtuvo la beca Fullbright-García Robles para estudiar el doctorado en The City University of New York (cuny), donde se especializó en cuestiones de autorregulación y evaluación educativa. Por más de 10 años estuvo como subdirectora del área de Trayectoria Escolar en la Dirección General de Evaluación Educativa trabajando temas vinculados al abandono y rezago escolar. Actualmente está a cargo de la Subdirección de Investigación en Educación de la Coordinación de Desarrollo Educativo e Innovación Curricular (CODEIC), Universidad Nacional Autónoma de México (UNAM).

\section{Maura Pompa Mansilla}

maurapompa@unam.mx

Licenciada en Pedagogía por la Facultad de Filosofía y Letras de la unAm. Ha trabajado desde hace más de 14 años en instituciones del sector educativo, ocho de los cuales fue investigadora en el Centro de Estudios Educativos A. C. (CEE). Se ha desempeñado primordialmente como investigadora en educación en temas relacionados con equidad, educación indígena, evaluación y desempeño docente, inter y multiculturalidad, identidad del profesorado, e inclusión de tecnologías de la información y la comunicación (TIC) en el aula. Cuenta con algunas publicaciones al respecto en libros y revistas. También ha impartido diversos cursos y talleres a nivel local y nacional sobre las temáticas antes señaladas, y participado en congresos en México y el extranjero. Actualmente es la Jefa del Departamento de Investigación Traslacional en Educación en la CODEIC, es docente de posgrado, e imparte diferentes contenidos en cursos y diplomados relacionados con la investigación.

\section{Flora Beatriz Hernández Carrillo}

florahc@unam.mx

Licenciada y doctorante en Psicología, por la Facultad de Psicología de la unAm. Realizó una estancia académica en la Facultad de Educación de la Universidad de Cambridge, en uk. Investigadora en los proyectos Aprendiendo Juntos en Educación Primaria, A tool for analysing dialogic interactions in classrooms y The role of dialogic a/symmetrical interactions among peers in diverse multimodal reading situations. Coautora del capítulo "Dialogical Interactions among peers in collaborative writing contexts", "The teacher scheme for educational dialogue analysis (T-SEDA)" e "Investigación traslacional en ciencias de la salud: implicaciones educativas y retos". Ha trabajado en el sector educativo y empresarial desde hace 13 años. Cuenta con experiencia como docente de educación básica, media superior y superior, así como instructor en industrias en México y el extranjero, en modalidades presenciales y a distancia. Investigadora en tópicos de psicopedagogía, métodos educativos, desarrollo profesional, evaluación de programas e interacción sectores educativo-productivo.

\section{Melchor Sánchez Mendiola}

melchorsm@unam.mx

Coordinador de Desarrollo Educativo e Innovación Curricular en la Universidad Nacional Autónoma de México (UnAM). Doctor en Ciencias, Educación en Ciencias de la Salud por la UNAM; maestro en Educación en Profesiones de la Salud por la Universidad de Illinois en Chicago, EUA. Médico pediatra por la Universidad del Ejército y Fuerza Aérea Mexicanos (UDEFA). Profesor de carrera titular C de tiempo completo definitivo en la División de Estudios de Posgrado de la Facultad de Medicina, unam. 
"Una experiencia de formación: la Educación basada en evidencias"

Magda Campillo Labrandero, Maura Pompa Mansilla,

Flora Beatriz Hernández Carrillo y Melchor Sánchez Mendiola

Vol. 20, Núm. 6, noviembre-diciembre 2019

Revista Digital Universitaria

"La actividad más alta que un ser humano puede alcanzar es aprender para entender porque el entendimiento es ser libre"

Baruch Spinoza

"La ciencia será siempre una búsqueda, jamás un descubrimiento real. Es un viaje, nunca una llegada"

Karl Popper

\section{Introducción}

El propósito del presente artículo es compartir a los lectores de la Roula experiencia de los cursos de Educación basada en evidencias (EBE) que la Coordinación de Desarrollo Educativo e Innovación Curricular (CODEIC) ofrece a los docentes de la Universidad Nacional Autónoma de México (unam) desde 2016, así como describir la conformación y actividades de la comunidad de práctica CEBE (Comunidad de Educación Basada en Evidencias). Tanto los cursos EBE como la comunidad son experiencias de formación docente novedosas que han vinculado el arte de la enseñanza con los conocimientos de la ciencia.

Idealmente, las decisiones para mejorar el proceso de enseñanzaaprendizaje deberían integrar los avances del conocimiento científico en educación. Durante este año en México, se realizaron cambios a los artículos constitucionales en materia educativa y se incorporó expresamente en nuestra Constitución el derecho humano a gozar de los beneficios del desarrollo de la ciencia y la innovación tecnológica. Así, este derecho universal, establecido desde 1948 en la Declaración Universal de los Derechos Humanos, tiene ahora una expresión formal en la Constitución mexicana. En este contexto, la EBE adquiere una gran relevancia, ya que busca que los docentes informen sus decisiones y acciones en los hallazgos encontrados en la literatura científica publicada en el campo educativo. Con los cursos EBE se espera actualizar y profesionalizar a los docentes universitarios para que puedan adecuarse a las necesidades educativas actuales.

\section{La formación docente}

De acuerdo con la Real Academia de la Lengua Española, la palabra formación, proviene del latín formatio -ōnis, y significa la acción y efecto de formar o formarse; a su vez, formar es un verbo transitivo que alude al acto de preparar intelectual, moral o profesionalmente a una persona o a un grupo de personas. Docente, por su parte, tiene un origen latino que es docens-entis, participio presente de docēre y refiere a enseñar; por tanto, se define como un adjetivo que indica que es perteneciente o relativo a la enseñanza. Para este texto entenderemos la formación docente como el acto de preparar profesionalmente al maestro que se dedica a enseñar. 
La formación de profesores tendría que ser un proceso permanente, donde se busque su profesionalización a fin de adecuarse a los cambios científicos y sociales de su contexto, del país y del mundo en una época caracterizada por la globalización y cambios constantes. Estos procesos de cambio impactan, sin lugar a dudas, la labor del profesor y la formación que recibe, e influyen en la valoración que la sociedad hace de su función. Lo anterior se acentúa aún más en los docentes adscritos a instituciones públicas de gran magnitud, como la UNAM (CODEIC-UNAM, 2018).

Un aspecto importante para fomentar la profesionalización de los docentes es que la formación y la actualización se den de forma equivalente para que los conocimientos disciplinares sean vigentes. Al mismo tiempo, los métodos de enseñanza deben ser apropiados para que el profesor pueda transmitir esos saberes a sus estudiantes. En ese sentido, si los estudiantes son la razón de ser de las instituciones educativas, los profesores son el componente primordial para que los alumnos alcancen sus objetivos académicos. Por lo tanto, es ineludible poner énfasis en que, además de poseer los conocimientos y habilidades disciplinarias, los docentes cuenten con una formación y actualización permanente en asuntos específicos, relativos y relevantes para la enseñanza en su campo disciplinario (Sánchez, Martínez y Hernández, 2019).

El cúmulo de cambios e innovaciones a los que está sujeta la profesión docente no siempre va acompañado de las adecuaciones necesarias en los procesos de formación y profesionalización. La Educación basada en evidencias (EBE) es una alternativa para atender algunos de estos vacíos, a la vez que contribuye a la toma de decisiones informadas relacionadas a los procesos educativos.

\section{¿Qué es la Educación basada en evidencias (EBE)?}

En 1996, el educador británico David Hargreaves, en una conocida conferencia titulada "La enseñanza como una profesión basada en la investigación: posibilidades y prospectiva", utilizó por primera vez el concepto de enseñanza basada en la investigación, que más tarde derivó en la enseñanza basada en la evidencia y posteriormente en lo que hoy se conoce como educación basada en evidencias. Aunque el origen de la práctica basada en evidencias surge de la medicina, la EBE en el ámbito educativo tiene un papel muy importante (Hederich, Martínez y Rincón, 2014).

La EBE busca que los docentes y otros profesionales involucrados en la educación informen sus decisiones y acciones con los hallazgos encontrados en la literatura científica publicada en el campo educativo. Su principal propósito es fortalecer el juicio profesional de los involucrados en el campo y la práctica educativa, a partir de la investigación científica (Kvernbekk, 2017). 
Figura 1. Pasos de la educación basada en evidencias.
Figura 2. Modelo funcional de la EBE (recuperado de Hederich et al., 2014).
Asimismo, esta práctica emplea un método sencillo, sistemático y ágil, que permite a los docentes aproximarse a los temas y estudios que resulten de apoyo y sustento para el ejercicio de su disciplina. Esto implica partir de preguntas de investigación específicas, así como realizar estrategias de búsqueda y análisis que faciliten identificar, evaluar y sintetizar la evidencia obtenida (ver figura 1). Este proceso de indagación no representa un seguimiento de pasos o reglas predefinidas o fijas, por el contrario, la EBE promueve la práctica de mecanismos que favorezcan las inferenciasy derivaciones de juicios profesionales sustentados en resultados confiables.

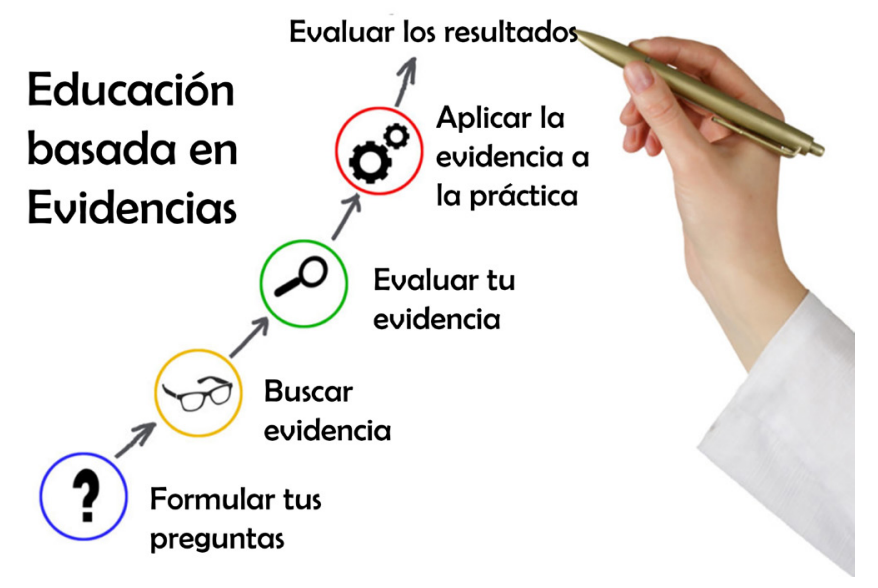

Uno de los elementos que definen una práctica profesional basada en la evidencia es la pretensión de que las decisiones profesionales cotidianas estén apoyadas en los resultados aportados por la investigación científica, así como de la experiencia y las necesidades de los involucrados. Por ejemplo, un modelo funcional EBE (Hederich, Martínez y Rincón, 2014) contempla que las decisiones educativas son un producto de la evidencia científica, la experiencia del profesor, los intereses y las necesidades del estudiante, así como de las interacciones que se den entre éstas (ver figura 2).

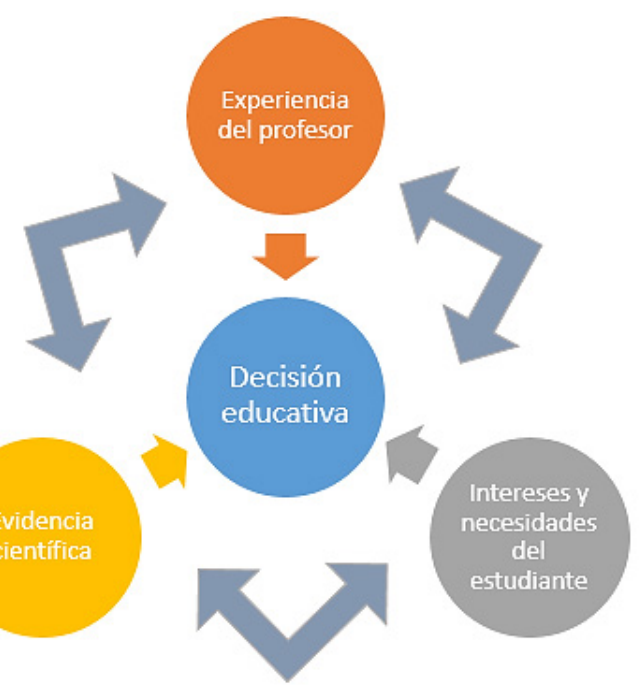


"Una experiencia de formación: la Educación basada en evidencias"

Magda Campillo Labrandero, Maura Pompa Mansilla,

Flora Beatriz Hernández Carrillo y Melchor Sánchez Mendiola Vol. 20, Núm. 6, noviembre-diciembre 2019

Revista Digital Universitaria

La cantidad de publicaciones propias de una disciplina puede ser abrumadora, por lo que una herramienta de la EBE son las revisiones sistemáticas, que integran, a partir de metodologías específicas, información sobre diversas publicaciones científicas de un tema en particular. Un evento crucial para la EBE en ciencias sociales fue la creación de la Campbell Collaboration, en honor al psicólogo norteamericano Donald Campbell, que surgió en 1999 con el fin de producir revisiones sistemáticas en educación, justicia, bienestar social y desarrollo internacional.

\section{Los cursos EBE}

Los cursos EBE se diseñan ante la necesidad de promover en la unAm un modelo práctico para toma de decisiones y acciones educativas, fundamentadas en la mejor evidencia científica disponible. En 2016, la coDElc implementó su primer curso. La meta es que los participantes trasladen los conceptos teóricos de la eBE a la aplicación práctica y que puedan implementar las herramientas que proporciona la EBE en su entorno de trabajo. Si bien el curso está dirigido a docentes de educación media superior y superior, también participan estudiantes, académicos y funcionarios universitarios.

Cada curso dura 20 horas y consta de dos sesiones presenciales de seis horas cada una, más tareas a distancia entre sesiones. En la primera jornada se discuten los conceptos esenciales de la EBE, se trabaja en cómo hacer preguntas de educación relevantes y cómo buscar la mejor evidencia en la literatura educativa. En la segunda se analiza cómo evaluar críticamente artículos de investigación en educación que utilizan metodologías cuantitativas, cualitativas o mixtas y meta-análisis o revisiones sistemáticas. Además, se realizan tareas colaborativas para conocer y negociar cómo aplicar la educación basada en evidencias en la práctica docente. Durante el trabajo a distancia los participantes realizan de manera individual diversas lecturas y hacen una revisión crítica de diferentes fuentes que contienen evidencias educativas.

Los objetivos del curso son que los participantes: 1) conozcan los conceptos esenciales en los que se sustenta la EBE, 2) planteen preguntas educativas relevantes, 3) hagan búsquedas bibliográficas efectivas, 4) identifiquen diferentes bases de datos en educación y 5) conozcan y apliquen criterios para evaluar la evidencia publicada en la literatura educativa.

La planta docente la conforman personal de la codelc y de la Dirección General de Bibliotecas de la UnAM. Para acreditar el curso se requiere que los participantes asistan a las dos sesiones presenciales y que completen el trabajo asignado. A los egresados se les extiende una constancia de asistencia con valor curricular.

LOS curSOS EBE se ofertan periódicamente en la UNAM, como parte del programa de Educación Continua de la codeic. Desde 2016 a la fecha se han ofertado ocho cursos y atendido a 177 participantes: 
Figura 3. Evaluación de los cursos de Educación basada en evidencias en la unam, durante los años 2016 y 2017 (formato inicial).

\begin{tabular}{|c|c|c|c|c|c|c|c|c|}
\hline \multicolumn{9}{|c|}{ Años } \\
\hline & 2016 & \multicolumn{3}{|c|}{2017} & 2018 & & \multicolumn{2}{|c|}{2019} \\
\hline Emisión & 1 & 2 & 3 & 4 & 5 & 6 & 7 & 8 \\
\hline Fechas & Octubre & $\begin{array}{l}\text { Marzo- } \\
\text { abril }\end{array}$ & Agosto & $\begin{array}{l}\text { Noviembre- } \\
\text { diciembre }\end{array}$ & Abril & Octubre & Marzo & Mayo \\
\hline $\begin{array}{l}\text { Participantes } \\
\qquad(\mathrm{N}=177)\end{array}$ & 22 & 26 & 32 & 20 & 15 & 24 & 20 & 18 \\
\hline
\end{tabular}

En estos cuatro años, cerca de $70 \%$ de los participantes han sido mujeres, la mitad de los asistentes cuenta con licenciatura y una tercera parte con maestría o especialidad. La cuarta parte labora en el bachillerato y $31 \%$ en alguna facultad. La mayoría imparte clases en licenciatura (37\%) y en bachillerato (27\%).

Los cursos han sido muy bien recibidos por los docentes. Se hicieron dos formatos de evaluación, el primero se utilizó en 2016 y 2017, el segundo en 2018 y 2019. En ambos se evaluaron el cumplimiento de los objetivos, la pertinencia de los materiales y de los contenidos, la preparación y la calidad de los instructores y la gestión administrativa. En todos los componentes de la evaluación las calificaciones fueron muy altas (ver figuras 3 y 4). Prácticamente $100 \%$ de los docentes dijo que recomendaría el curso a otros colegas y que el curso les permitiría aplicar esta metodología.

Para dar continuidad a los contenidos de los cursos e intercambiar experiencias sobre su aplicación se creó la comunidad de prácticas EBE, que se describe en la siguiente sección.

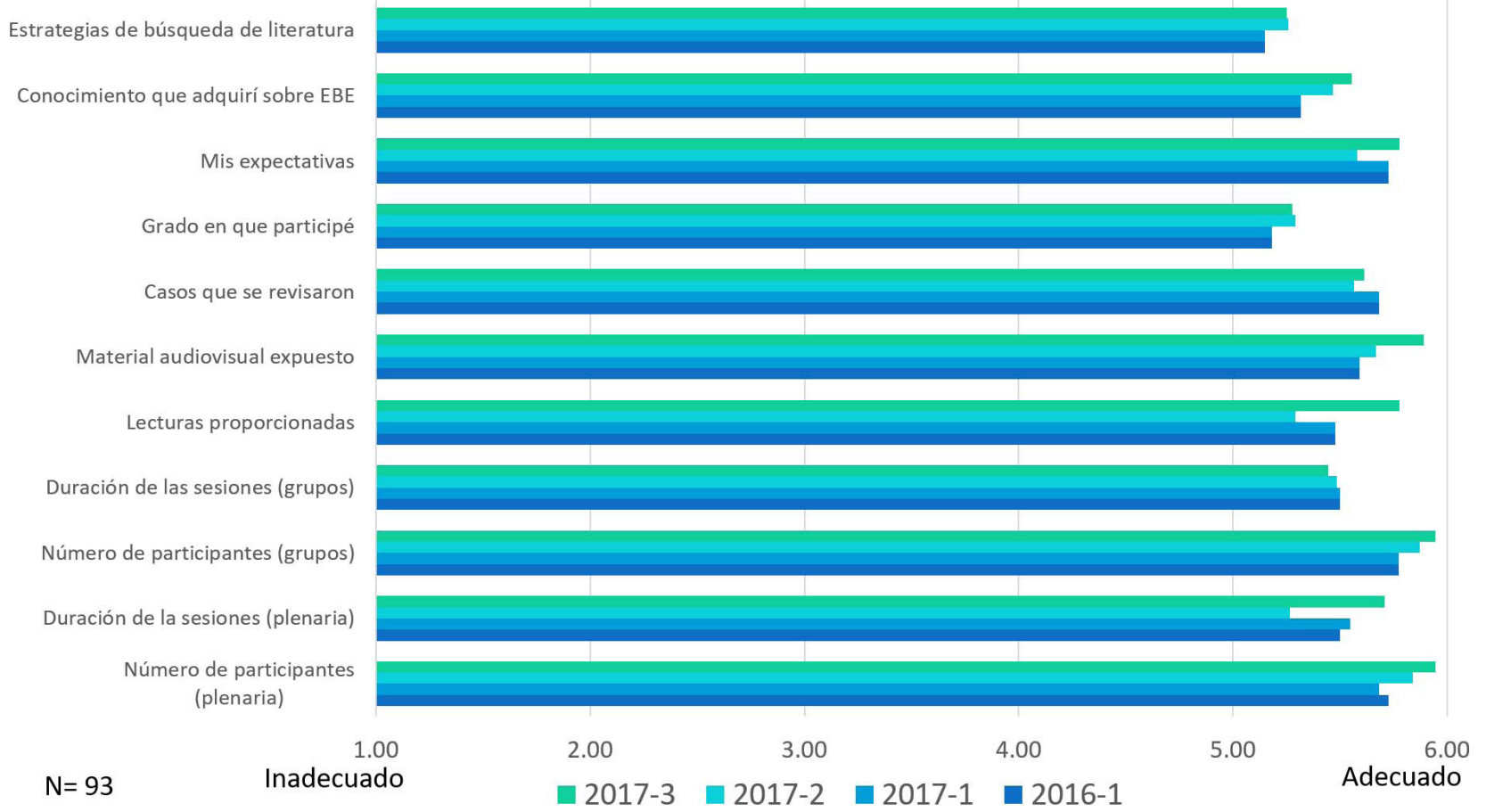


"Una experiencia de formación: la Educación basada en evidencias"

Magda Campillo Labrandero, Maura Pompa Mansilla,

Flora Beatriz Hernández Carrillo y Melchor Sánchez Mendiola

Vol. 20, Núm. 6, noviembre-diciembre 2019

Revista Digital Universitaria

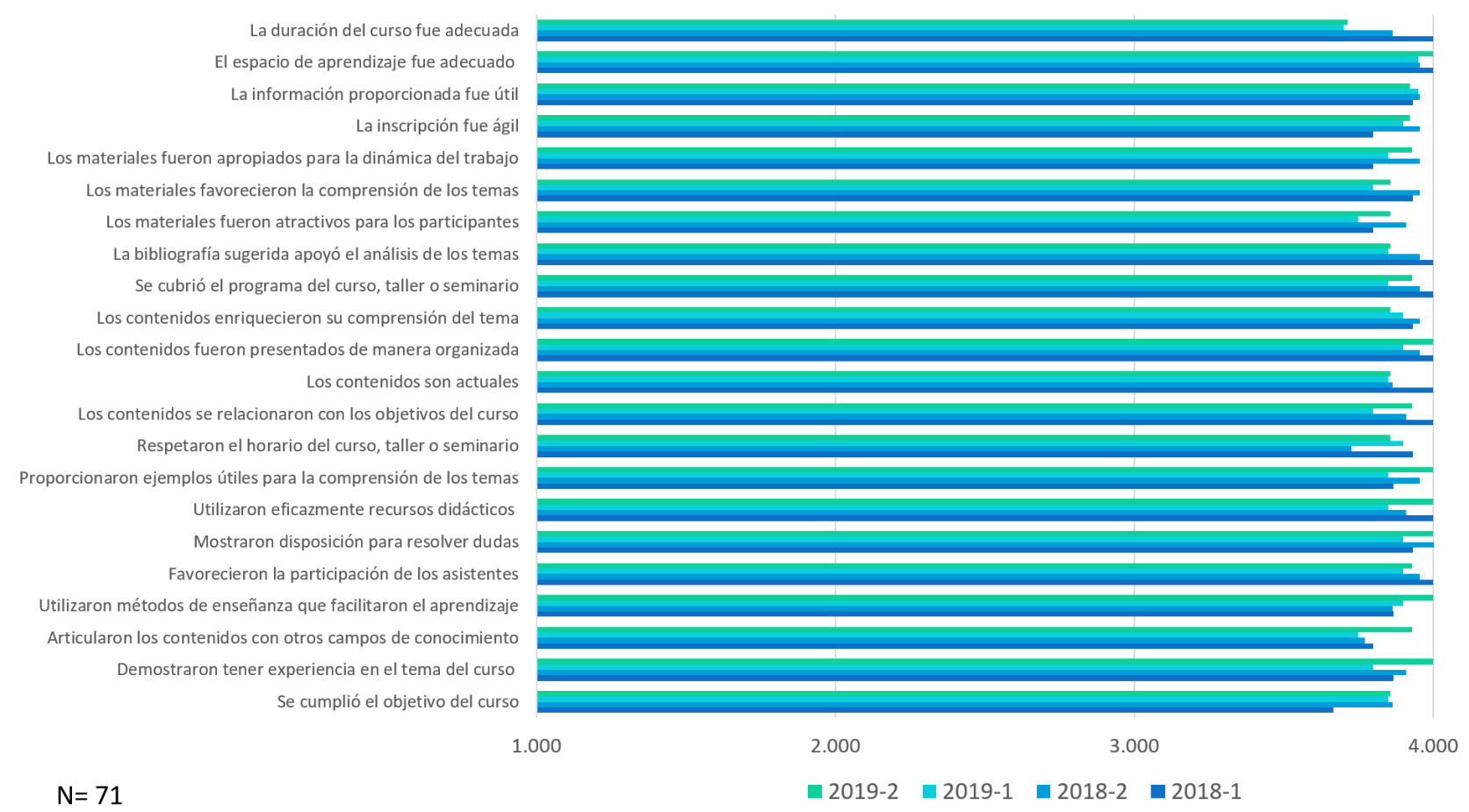

Figura 4. Evaluación de los cursos de Educación basada en evidencias en la unam, durante los años 2018 y 2019 (formato actual).

\section{CEBE}

La Comunidad de Educación Basada en Evidencias (севE) es, hasta donde pudieron identificar los autores, la primera comunidad virtual de este tipo en Latinoamérica. Se diseñó y construyó en 2018 como una comunidad de práctica con los egresados de los cursos EBE, con el fin de promover la educación basada en evidencias, así como las experiencias en su uso e implementación.

La comunidad cuenta con una plataforma en línea (www.cebe.unam.mx) y una red social virtual; periódicamente se organizan encuentros presenciales. Los miembros realizan actividades para promover la movilización y el intercambio de evidencias que respalden la toma de decisiones educativas sustentadas en la práctica de la EBE.

Las prácticas de la comunidad son desarrolladas y adaptadas a partir de un compromiso compartido (Wenger, 1998). Para ello, se llevan a cabo actividades a distancia y presenciales, diseñadas para propiciar la interacción entre miembros, y fundamentadas en el aprendizaje basado en la experiencia o en la construcción de relaciones efectivas entre pares (Steinert et al., 2016).

La CEBE Cuenta con roles de promotores-gestores, que son los miembros a cargo de la gestión de actividades de la comunidad por períodos determinados; personal de la coDelc brinda soporte en el diseño, realización y valoración de las actividades de la comunidad, y los miembros egresados del curso EBE. El sitio web se divide en cinco secciones: identidad, actividades, miembros, experiencias y red social. 
"Una experiencia de formación: la Educación basada en evidencias"

Magda Campillo Labrandero, Maura Pompa Mansilla,

Flora Beatriz Hernández Carrillo y Melchor Sánchez Mendiola Vol. 20, Núm. 6, noviembre-diciembre 2019

Revista Digital Universitaria

Otras comunidades internacionales que buscan vincular el quehacer cotidiano con el método de la EBE son Empirical Educator y el Center for Research and Reform in Education, en EUA, y la comunidad de MESHGuides, en Inglaterra. De forma paralela, en América Latina ya se están gestando otras estrategias tales como el mooc Qué funciona en educación: políticas educativas basadas en evidencia, diseñado por el Banco Interamericano de Desarrollo.

\section{Reflexiones finales}

Las decisiones que como docentes tomamos en el aula se basan, en muchos de los casos, en nuestros propios dogmas, tradiciones gremiales, intuición, recomendaciones de expertos o colegas, aprendizajes en congresos, normatividad institucional, o bien, a través de búsquedas en diversas fuentes como internet o acervos bibliográficos. Aunque todos estos elementos influyen, la experiencia compartida en el texto ilustra que, a través de los cursos EBE, se puede profesionalizar la práctica docente para que los maestros incorporen los resultados de la investigación científica en sus decisiones educativas.

Integrar la experiencia docente junto con las necesidades de los estudiantes y el cúmulo de la evidencia como resultado de la investigación científica requiere combinar el arte fino de la enseñanza con el rigor metodológico de las ciencias, todo ello, con el fin de buscar y descubrir nuevas formas de mejorar nuestra práctica.

Invitamos a nuestros lectores a explorar la Educación basada en evidencias, a conocer la página de la comunidad.

\section{Referencias}

* Coordinación de Desarrollo Educativo e Innovación Curricular (2018). Centro de Formación y Profesionalización Docente de la UNAM, Fundamentación [Documento de trabajo no publicado]. México. Universidad Nacional Autónoma de México, Secretaría General, Coordinación de Desarrollo Educativo e Innovación Curricular.

* Hargreaves, David (1996). Teaching as a research based profession: Possibilities and prospects. The Teacher Training Agency. Annual Lecture. Recuperado de: https:// eppi.ioe.ac.uk/cms/Portals/0/PDF\%20reviews\%20and\%20summaries/TTA\%20 Hargreaves\%20lecture.pdf.

* Hederich, C., Martínez, J. y Rincón, L. (2014). Hacia una educación basada en la evidencia. Revista Colombia de Educación, 66, 19-54.

* Kvernbekk, T. (2019). Evidence-Based Educational Practice. En Oxford Research Encyclopedia of Education (pp. 1-20). Recuperado de: https://doi.org/10.1093/ acrefore/9780190264093.013.187.

- Lave, J. y Wenger, E. (1991). Situated Learning. Legitimate peripheral participation. Nueva York: Cambridge University Press. 
* Marland, M. (1985). El arte de enseñar (Técnicas y organización del aula). Madrid, España: Ediciones Morata.

* Moretti, G. y Alessandrini, G. (2015). Community of Practice and Teacher's Professional Development: An Explorative Survey. Journal of Educational, Cultural and Psychological Studies, 11, 253-273.

* Philpott, C. y Poultney, V. (2018). Evidence-based Teaching. Reino Unido: Critical Publishing

* Real Academia Española (2018). Diccionario de la lengua española, [23. ${ }^{a}$ ed., versión 23.2 en línea]. Recuperado de: https://dle.rae.es.

* Sánchez Mendiola, M., Martínez Hernández, A. M. del P. y Hernández Romo, A. K. (2019). ¿Por qué es fundamental un proyecto de formación y profesionalización docente en la Universidad Nacional Autónoma de México? En M. Sánchez y M. P. Martínez (eds.), Formación Docente en la unam: Antecedentes y la voz de su profesorado (pp. 15-29). Ciudad de México, México: unam.

* Steinert, Y., Mann, K., Anderson, B., Barnett, B., Centeno, A., Naismith, L., Prideaux, D., Spencer, J., Tullo, E., Viggiano, T., Ward, H. y Dolmans, D. (2016). A systematic review of faculty development initiatives designed to enhance teaching effectiveness: A 10-year update: BEmE Guide No. 40. Medical Teacher, 38 (8), 769786.

* Wenger, E. (1998). Comunidades de práctica. Barcelona: Paidós

\section{Agradecimientos}

Agradecemos al equipo docente que a lo largo de las ocho emisiones de los cursos EBE ha colaborado en la planeación e implementación de los cursos, al personal administrativo de la CODEIc, así como al área de Educación Continua por todo su apoyo en la gestión y en la logística. Gracias a la colaboración de estos actores la exitosa emisión de estos cursos ha sido posible.

\section{Cómo citar este artículo}

* Campillo Labrandero, Magda, Pompa Mansilla, Maura, Hernández Carrillo, Flora Beatriz y Sánchez Mendiola, Melchor (2019). Una experiencia de formación: la Educación basada en evidencias. Revista Digital Universitaria (RDU). Vol. 20, núm. 6 noviembre-diciembre. Dol: http://doi.org/10.22201/codeic.16076079e.2019.v20n6.a6.

Recepción: 17/10/2019. Aprobación: 23/10/2019 